\title{
CONFERENCIA
}

\section{PRÁCTICA BASADA EN EVIDENCIAS: Futuro de la Motricidad Orofacial}

Irene Marchesan ${ }^{1}$

La Motricidad Orofacial (MO) es una especialidad reciente en la Fonoaudiología mundial. Una de las razones de esta especialidad de ser menos conocida en el mundo se debe al echo de que, hasta el año 2000, no se tenia aún una definición específica de esta especialidad en si, y también, no tenia bien definido cual seria su campo de acción. Esa falta de definición continuaba ocurriendo, a pesar que el Consejo Federal de Fonoaudiología ya tenia creado, en 1996, una resolución que instituye cuatro grandes áreas de conocimiento, siendo una de ellas la MO. Solamente en 2001, el Comité de MO de la Sociedad Brasilera de Fonoaudiología publico el primer documento, considerado oficial de la MO, el cual contenía la definición de la MO y lo que era relevante correspondiente a su ámbito de conocimiento. En la medida en que esa especialidad en el Brasil creo la definición de lo que era la $\mathrm{MO}$, y delimito su campo de trabajo, hubo un mayor número de publicaciones en formato de libros o capítulos de libros. Esas publicaciones fueron aumentando vertiginosamente y hasta sin control. Todo lo que era realizado en las clínicas fue descrito y publicado de alguna forma.

Para que la especialidad fuese reconocida como una ciencia, donde sus resultados fuesen comprobados, los cursos de Pos-Grado existentes en maestría y doctorado comenzó a desarrollar proyectos en el área de la MO. Siendo así, que los Estudiantes de maestría y doctorado implicados específicamente en ese campo de trabajo, comenzaron a desarrollar investigaciones controladas sobre lo que hacen. De ahí en adelante, tales trabajos pasaron a ser publicados en forma de artículos, y ya no de libros o capítulos de libros. Estudios clínicos controlados basados en evidencias fueron realizados en diferentes centros de excelencia en el Brasil.

El hecho de que existieran publicaciones, maestrías y doctorados con enfoque específico en la especialidad de $\mathrm{MO}$ hizo que la especialidad creciese y se profundice cada vez más en los diversos campos abordados por la MO. Como la definición de la MO dijo que esa área seria el campo de la Fonoaudiología dedicado para el estudio/investigación, prevención, evaluación, diagnóstico, desarrollo, capacitación, mejoramiento y rehabilitación de los aspectos estructurales y funcionales de las regiones orofacial y cervical, se puede pronosticar el gran número de publicaciones que llegaron a existir en cada tema abordado por esta especialidad. Cuanto más un área crece, un número mayor de investigaciones viene a existir para demostrar su efectividad en los tratamientos propuestos. A partir de ahí, terapeutas, profesores e investigadores del área de la $\mathrm{MO}$ en otros países, también desarrollaron más y más la MO en sus Universidades. Todo eso nos lleva a creer que el futuro de la MO estará garantizado desde que los hallazgos clínicos sean probados por investigaciones bien delineadas y realizadas dentro de los conceptos reconocidos mundialmente como ciencia. O sea, las prácticas desarrolladas en las clínicas, aunque tengan eficacia y eficiencia,

1. Doutorado em Educação pela UNICAMP; Mestre em Distúrbios da Comunicação pela PUC-SP; Especialista em Motricidade Orofacial Título 01/1996; Fonoaudióloga Clínica desde 1978.

Atual Presidente da Sociedade Brasileira de Fonoaudiologia Gestão 2014-2016; Publicações de livros, capítulos de livros e artigos na área da Motricidade Orofacial (MO). 